ISSN e- 2594-1100

en-claves del pensamiento / núm. 30 / julio-diciembre / 2021 / e422

https://doi.org/10.46530/ecdp.v0i30.422 ARTÍCULOS

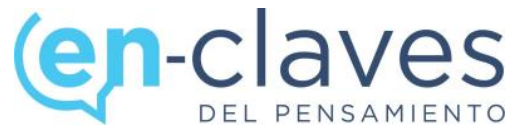

\title{
EL TRASFONDO ONTOLÓGICO DE LA MODERNIDAD OCCIDENTAL: REVISIÓN CRÍTICA DE LA ESCISIÓN NATURALEZA/CULTURA
}

\author{
The Ontological Background of Western Modernity: \\ A Critical Review of Nature/Culture Dualism
}

\begin{abstract}
Vanessa Ivana Monfrinotti Lescura, Universidad Nacional del Comahue, Argentina
Correo electrónico: vanee.ml@hotmail.com
\end{abstract}

Recibido: 08/06/2020

Aceptado: 10/02/2021

Publicado: 01/07/2021

\begin{abstract}
Resumen. En el presente artículo se realiza un recorrido conceptual en torno a la conformación del dualismo naturaleza/cultura en el marco de una ontología específica: la ontología dualista propia de la modernidad occidental. En una primera instancia, las raíces de dicho binomio remiten al escenario del siglo XVII, enunciado filosóficamente por René Descartes a partir de la escisión res cogitans/res extensa. Este proceso no finaliza allí, por lo que, en este trabajo, se da cuenta del modo en que dicho dualismo requirió de complejos mecanismos (epistémicos, filosóficos, culturales, históricos, políticos, etc.) que cristalizaron la conformación de dos ámbitos ontológicamente autónomos, por un lado, lo socio-cultural y, por otro, lo natural. El propósito de este análisis es dar cuenta de cuáles son las bases ontológicas que nos condujeron a un presente inmerso en una crisis civilizatoria, anunciada así por diversas posturas epistémicas y movimientos sociales.
\end{abstract}

Palabras claves: ontología dualista, modernidad, naturaleza/cultura, ontología política, naturalismo.

Abstract. In this paper, a conceptual review is made around the conformation of nature/culture dualism, in their insertion on dualist ontology of the western modernity. In the first place, the roots of this binomial refer to the 17th century scenario, philosophically uttered by René Descartes in the separation between res cogitans/res extensa. This process does not end there, so, in this text, we realize the way in which such dualism required complex mechanisms (epistemic, philosophical, cultural, historical, political, etc.) that crystallized the conformation of two ontologically autonomous spheres, on one hand, the socio-cultural and, on the other, the natural. The purpose of this analysis is to give an account of the ontological bases that led us to a present immersed in a crisis of civilization, thus announced by different epistemic positions and social movements.

Keywords: Dualistic ontology, modernity, nature/culture, political ontology, naturalism.

Cómo citar: Monfrinotti, V, (2021). El trasfondo ontológico de la modernidad occidental: revisión crítica de la escisión naturaleza/cultura. En-claves del pensamiento, 0(30), e422. doi: https://doi.org/10.46530/ecdp.v0i30.422

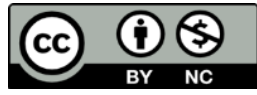

Esta obra está protegida bajo una Licencia

Creative Commons Atribución - No comercial

4.0 Internacional

www.enclavesdelpensamiento.mx

https://doi.org/10.46530/ecdp.v0i30.422 


\section{Preludio: sobre la necesidad de revisar la ontología dualista moderna}

Una de las cuestiones que se torna urgente a la luz de la crisis civilizatoria actual es la pregunta por las premisas ontológicas que han formado parte de la modernidad capitalista hasta nuestros días. Tal pregunta asume la coimplicación entre ontologías y política, así como también entre episteme y política. ${ }^{1}$ En este sentido, el análisis que desplegamos se focaliza en la ontología dualista moderna, cuyas consecuencias devastadoras ponen en riesgo la continuidad de la vida humana y no-humana en nuestro presente. Por lo que se trata de un recorrido filosófico-conceptual que tiene en cuenta la inserción de dicha ontología en la trama del capitalismo moderno/colonial. ${ }^{2}$

Este trabajo atiende a las bases ontológicas que aún hoy persisten y que sostienen un modelo de expoliación y apropiación de los mundos que se han delimitado como fuera de "lo humano", en el marco del capitalismo moderno/colonial. El trasfondo ontológico al que referimos (que configura lo antropólogo, lo epistemológico, lo político, lo cultural, etc.) tiene larga data en el pensamiento occidental y es enunciado por René Descartes en el contexto del siglo XVII. Ahora bien, a pesar de encontrar la expresión emblemática de la ontología dualista moderna en la tradición cartesiana, dicha ontología se desenvuelve

\footnotetext{
${ }^{1}$ En ello coinciden un conjunto de planteos, entre los que se encuentra el giro decolonial, las propuestas de la ontología política y políticas de la ontología. Cfr. María Eugenia Borsani, "El encuentro con la decolonialidad desde este sur", Ejercicios decolonizantes en este sur (Buenos Aires: Ed. del Signo/Duke University, 2014); y Arturo Escobar, Autonomía y diseño: la realización de lo comunal (Popayán: Editorial Universidad del Cauca, 2016).

${ }^{2}$ Cabe señalar que la designación "moderno/colonial" remite a la perspectiva epistémico-política del giro decolonial, según la cual, a la modernidad le es inherente el despliegue de la colonialidad. Cfr. Aníbal Quijano, "Colonialidad y modernidad-racionalidad", en Zulma Palermo y Pablo Quintero, Aníbal Quijano. Textos de fundación (Buenos Aires: Ed. del Signo / Duke University, 2014); Walter Mignolo, Desobediencia epistémica. Retórica de la modernidad, lógica de la colonialidad y gramática de la descolonialidad (Buenos Aires: Ed. del Signo / Duke University, 2010); y Arturo Escobar, "Mundos y conocimientos de otro modo", Revista Tabula Rasa, núm. 1 (enero-diciembre 2003): 51-86. Se entiende por "la inserción de dicha ontología en la trama del capitalismo moderno/colonial" como una explicitación de la relación entre la ontología moderna y el despliegue del capitalismo, a fin de dar cuenta del interés que cobra la temática para quien escribe y no de un objetivo a desarrollar en artículo. Consideramos que la revisión de las principales notas de la ontología moderna canónica, adquiere sentido a la luz de la inquietud de cómo llegamos a la crisis civilizatoria actual y qué ontología ha dado lugar a qué prácticas. Para trabajos futuros se profundizará en la lógica de separación y de subyugación que reviste tal ontología dualista, es decir, el despliegue de su colonialidad que ha avanzado con el borramiento y el silenciamiento históricos de mundos otros, así como también sus consecuencias en términos de devastación territorial.
} 
hasta nuestros días conformando una de las características propias de la episteme moderna en su conjunto y de nuestra cultura occidental.

Así, se pretende dar cuenta de los múltiples mecanismos que han fortalecido la escisión entre naturaleza y cultura, es decir, la instauración de un dualismo ontológico que separa de manera abismal al hombre y la naturaleza. Éste se repliega sucesivamente fundando otros dualismos, entre los cuales encontramos sujeto/objeto, mente/cuerpo, varón/mujer, civilización/barbarie, Nosotros/Ellos, occidente/oriente, humano/no-humano, entre otros. En este sentido, coincidimos con María Eugenia Borsani cuando señala que:

La ontología occidental se constituyó sobre un tipo de pensamiento, que [...] denominé 'pensamiento opositivo', aquel que piensa el mundo en términos de contrastes y binarismos que es propio de nuestro acervo cultural occidental. Dicho entrenamiento ontológico divide el mundo en pares opositivos excluyentes, pensamiento binario propio de la episteme moderna. ${ }^{3}$

De este modo, advertimos la lógica opositiva y excluyente que ha operado en el modo de comprender el mundo y la relación jerárquica que el sujeto moderno (hombre, occidental, blanco, burgués) ha establecido con el "mundo natural". La conformación ontoepistémica de la modernidad ha determinado los modos de ser, hacer y conocer que han predominado y se torna necesario revisarlos bajo la clave de la ontología política. Bajo una mirada crítica a la racionalidad moderna, se muestra en su condición antropocéntrica, logocéntrica, androcéntrica, instrumental, monológica y colonial.

Es así que, desde las perspectivas críticas de la Modernidad/Colonialidad, ${ }^{4}$ se advierte la relación de la actual crisis planetaria con una ontología de devastación inherente a la modernidad occidental. Por ende, se hace menester un desplazamiento de aquella racionalidad dualista y los consecuentes modelos de pensamiento que se desprenden de ella, habida cuenta de la imposibilidad de responder ante la coyuntura planetaria y de diseñar mundos otros, dentro de las lógicas de pensamiento modernas. Esta urgencia se advierte en el carácter de fuerza geológica que adquiere la humanidad, a la luz de los recientes debates del Antropoceno, en tanto nueva era geológica.

\footnotetext{
${ }^{3}$ Borsani, "El encuentro", 35.

${ }^{4}$ Edgardo Lander, "Los límites del planeta y la crisis civilizatoria", Revista Venezolana de Economía y Ciencias Sociales, núm. 1 (enero-abril, 2011): 141-166.
} 
Si bien este artículo no profundizará en la compleja crisis ontológica y civilizatoria en la que nos vemos sumidos, ${ }^{5}$ proponemos en esta ocasión comprender cuál es la ontología que habitamos para poder diseñar y disoñar ${ }^{6}$ modos otros de ser, hacer y conocer, al decir de Arturo Escobar. ${ }^{7}$ En este sentido, vienen a cuenta las palabras de Philippe Descola sobre el Antropoceno, acontecimiento que sintetiza de forma dramática la crisis civilizatoria:

Así, la humanidad en general no originó el Antropoceno, sino un sistema, un modo de vida, una ideología, una manera de darle sentido al mundo y a las cosas, que sedujeron y se extendieron cada vez más y de las cuales es necesario entender sus particularidades si queremos acabar con aquél y cambiar de rumbo para intentar evitar algunas de sus consecuencias más dramáticas. ${ }^{8}$

Entonces, ubicamos la mirada en aquella manera de darle sentido al mundo que nos condujo al Antropoceno, es decir, específicamente en la ontología dualista dominante, desde la cual se instauró una opción civilizatoria totalizante, hoy en crisis. Asumimos, en este sentido, una férrea convicción de que otros mundos son posibles, bajo la necesidad de un desplazamiento ontológico, pero antes debemos desandar las premisas ontológicas propias de la modernidad capitalista.

\section{Dualismo ontológico en el pensamiento cartesiano}

La dicotomía naturaleza/cultura, en función de la cual se ha separado el mundo natural, por un lado, y el mundo de la cultura y la sociedad por otro, remite al andamiaje conceptual de la Filosofía Moderna. A partir de la tradición cartesiana se configura la idea de naturaleza como dominio ontológico, autónomo y objetivo, es decir, con propiedades específicas anteriores e

\footnotetext{
${ }^{5}$ Temática a desarrollar en una próxima instancia, en continuidad con este trabajo.

${ }^{6}$ Escobar, Autonomía y diseño.

7 Arturo Escobar es un pensador colombiano, profesor de la Universidad de Carolina del Norte en Chapel Hill, cuyas áreas de interés son la Ecología Política, los movimientos sociales, las discusiones sobre el posdesarrollo y los estudios sobre los "diseños para las transiciones". Su pensamiento es una puerta a la relacionalidad, la comunalidad y la pluriversalidad, es decir, a la reflexión crítica de la ontología dualista y la apertura hacia ontologías relacionales y múltiples.

${ }^{8}$ Philippe Descola, “¿Humano, demasiado humano?”, Desacatos, núm. 54 (mayo-agosto, 2017): 18.
} 
independientes a cualquier operación de conocimiento. La naturaleza se constituye como un campo posible de ser explicado por leyes y experimentado, concepción legitimada a su vez con la revolución científica del siglo XVII. Esta última es de gran relevancia, dado que a partir de la misma se comenzaron a trazar los lineamientos de una nueva forma de conocimiento que busca su legitimación por fuera de todo principio de autoridad (social, religiosa, etc.). Por tanto, también será de interés presentar algunas ideas sobre el método científico incipiente y su vínculo instrumental con lo que llamamos "naturaleza".

Lecturas usuales de la historia de la filosofía señalan a Descartes como el filósofo emblemático del comienzo de la filosofía moderna. Su pensamiento inauguró una nueva forma de hacer filosofía que colocó al hombre en el centro, a partir de una experiencia del yo en la que se fundó la subjetividad moderna, expresada en la primera certeza, el cogito. Su interés primordial, radicaba en fundar los cimientos del edificio del conocimiento sólo a partir de aquellas certezas que se pueden obtener del entendimiento y desechar todo aquello que provenga de nuestros sentidos, dado que no son fuente de conocimiento confiable para el racionalismo. Es así que en el conjunto de sus reflexiones esbozó los principios y los fundamentos de un nuevo modo de conocer el mundo, que de manera incipiente tramaban el camino de la ciencia y la técnica modernas.

En la filosofía cartesiana se hallan los rasgos principales de una lógica dualista que se asienta en el pensamiento y la cultura occidental, dominante hasta nuestros días. ${ }^{9}$ El dualismo que adquirió fuerza con Descartes se basó en la distinción de dos sustancias de naturaleza contraria, el alma ${ }^{10}$ y el cuerpo, la res cogitans y la res extensa, de las que da cuenta en sus obras. Respecto del primer término de este dualismo, la res cogitans es la primera certeza a la que arribó progresivamente con la aplicación de la duda metódica en su libro Meditaciones metafísicas. ${ }^{11}$ Ésta consiste en la certeza de saberse una cosa que piensa, aun cuando se encuentre dudando de todo y, de este modo, se le presentó como evidente la existencia de una sustancia pensante, formulada como cogito ergo sum o "pienso, luego existo". El mismo acto de pensar alcanza un estatus privilegiado,

\footnotetext{
${ }^{9}$ Es posible indagar en los antecedentes de esta lógica dualista en la Filosofía Antigua, tal es el caso de Parménides (ser/no ser) o de Platón (alma/cuerpo, epistemeldoxa, lo sensible/lo inteligible, etc.). Así también, se observa la continuidad de estas escisiones en la tradición judeo-cristiana.

${ }^{10}$ Entiéndase por alma "conciencia”, "espíritu”, “mente", "entendimiento".

${ }^{11}$ René Descartes, Meditaciones metafísicas (Buenos Aires: Prometeo, 2009).
} 
fundamento de la existencia del yo, de manera tal que el pensamiento se constituye como el atributo primordial de la conciencia; en palabras de Descartes: "es un atributo que me pertenece, el único que no puede ser separado de mí". ${ }^{12}$

Junto con la evidencia de la existencia de una sustancia pensante, Descartes afirmó que la misma es una sustancia distinta del cuerpo. Es decir, además de ser un conocimiento que precede al del cuerpo y que es más evidente, su existencia es independiente de cualquier cuerpo, del cuerpo humano y de las cosas materiales del mundo. Esta autonomía queda explicitada cuando el pensador escribe que "manifiestamente conocemos que para ser no tenemos necesidad de extensión, de figura, de ser en algún lugar, ni de ninguna otra cosa semejante que se pueda atribuir al cuerpo, y manifiestamente conocemos que nosotros somos en razón sólo de que pensamos". ${ }^{13}$ Entonces, la existencia del hombre como sustancia pensante es independiente ontológicamente de toda materialidad, corporalidad o res extensa. En este sentido, el sujeto (moderno) no le debe nada al resto de las sustancias que conforman el mundo, en tanto el fundamento de su existencia se encuentra en sí mismo, existe independientemente de las relaciones que pueda establecer con ese mundo.

El siguiente fragmento del Discurso del método da cuenta de separación de dos realidades independientes mutuamente:

[C]onocí por ello que yo era una sustancia cuya esencia y naturaleza toda es pensar, y que no necesita, para ser, de lugar alguno, ni depende de cosa alguna material; de suerte que este yo, es decir, el alma por la cual yo soy lo que soy, es enteramente distinta del cuerpo y hasta más fácil de conocer que éste, $y$, aunque el cuerpo no fuese, el alma no dejaría de ser cuanto es. ${ }^{14}$

En relación a la res extensa, el otro término del dualismo presentado en esta metafísica de la sustancia, refiere al atributo principal de las cosas materiales. La naturaleza del cuerpo o de la materia no se obtiene a partir de las cualidades que afectan a los sentidos (peso, color, dureza, etc.), sino de aquello que conocemos clara y distintamente por el entendimiento, que es la extensión en longitud, latitud y profundidad. ${ }^{15}$ En este punto, se

\footnotetext{
${ }^{12}$ Ibid., 50.

${ }^{13}$ René Descartes, Los principios de la filosofía (Barcelona: Alianza Editorial, 1995), 25-26.

${ }^{14}$ René Descartes, Discurso del método (Madrid: Editorial Gredos, 2011), 124.

${ }^{15}$ Cfr. Ibid., 74.
} 
hace manifiesta la visión mecanicista del mundo físico concebido como una gran máquina compuesta por piezas extensas en movimiento.

Cabe recordar que en la filosofía cartesiana también se postuló la existencia de una tercera sustancia, primordial en el orden del ser y creadora de las otras dos sustancias, la res infinita pensante (segunda certeza), es decir, Dios. No obstante, los atributos principales que puede tener una sustancia son dos, el pensamiento y la extensión. Tanto el yo como el mundo dependen de Dios para existir, pero son mutuamente independientes, son realidades diferenciadas entre ellas, autónomas. Sin embargo, en el marco filosófico cartesiano, la naturaleza humana está caracterizada por el encuentro de estas dos sustancias que conviven en el hombre, dando lugar a un dualismo antropológico. ${ }^{16}$

Este dualismo antropológico, que hace de la naturaleza humana un particular compuesto de mente-cuerpo, separa al hombre del resto de mundo, comprendido éste como un conjunto de cosas corpóreas, desprovistas de res cogitans. Dada esta escisión Descartes debió recurrir a la existencia de Dios para obtener certeza de la existencia del mundo y las cosas materiales, en otras palabras, iniciar el camino que lleva de la certeza de Dios al conocimiento de la realidad de las cosas y el mundo. Tal problema filosófico, que conduce a recurrir a Dios para salir del solipsismo, emerge a partir de la consolidación de un yo cartesiano desconectado ontológicamente del mundo, cuya existencia no tiene ningún vínculo necesario con el mundo físico, le es ajeno. Esto queda explicitado en el inicio de la cuarta meditación: "Y me parece que ya descubro un camino que me conducirá de esta contemplación del verdadero Dios [...] al conocimiento de las otras cosas del Universo". ${ }^{17}$

\section{El concepto mecanicista de la naturaleza y la matematización del universo}

Para explicar el mundo y dar cuenta de la naturaleza, incluida la del cuerpo humano, Descartes se sirvió de la metáfora de la máquina. Esta interpretación física del mundo es conocida como "mecanicismo" y es asumida por un conjunto de filósofos y científicos de los siglos XVII y XVIII. Dentro de este esquema, la naturaleza tiene un funcionamiento

\footnotetext{
${ }^{16}$ Cabe señalar que este punto se vuelve problemático porque después de haber separado radicalmente dos realidades, Descartes debe dar cuenta de la existencia de ambas en un mismo individuo. Es así que postula la presencia de la glándula pineal para conectar la mente con sus órganos sensoriales y el cuerpo. No amerita un desarrollo minucioso de esta problemática a la luz de los propósitos de este trabajo.

${ }^{17}$ Descartes, Meditaciones metafísicas, 75.
} 
automático al estar sujeta a leyes mecánicas que le aportan regularidad y hacen posible su conocimiento por medio de la física, haciendo de ella un objeto sometido a la explicación matemática. ${ }^{18}$ En este sentido, existe un vínculo directo con la indagación metafísica de las sustancias, que da cuenta de la extensión como atributo principal de los cuerpos, dado que es un atributo cuantificable. En tanto que la res extensa es medible, cuantificable, regulable y posee un funcionamiento mecánico, Descartes coincidía con Galileo Galilei en el mismo siglo, sobre la afirmación de que la naturaleza estaba escrita en el lenguaje de las matemáticas. La concepción esbozada sobre la naturaleza corporal se basa en el modelo en las matemáticas como fundamento para la obtención de conocimiento seguro.

La llamada res extensa corresponde al mundo físico-natural, ${ }^{19}$ entendido como el dominio compartido por los humanos y los animales (y, en general, por toda cosa material), el cuerpo. Por el contrario, la conciencia o la res cogitans, es caracterizada como el signo distintivo de la humanidad. La búsqueda permanente del rasgo de excepcionalidad de lo humano, ha gozado de reiterados intentos a lo largo del pensamiento occidental, singularidad que ha sido comprendida como privilegio ontológico. $^{20}$ A pesar de que la concepción mecanicista de la naturaleza en la época del racionalismo cartesiano no tiene una relación dicotómica con las nociones de "cultura" o "sociedad", sí aparece diferenciada de una naturaleza humana, como punto fijo indispensable para aprehender las regularidades de la naturaleza.

En función de lo desarrollado hasta aquí, se puede señalar la presencia de un esquema dualista que recorre el pensamiento cartesiano en diferentes niveles. Así, se observa un primer dualismo, de carácter metafísico, que clasifica y separa la realidad en dos sustancias, pensante y extensa. En segundo lugar, en un nivel antropológico, se encuentra la presencia de esta dualidad al interior del hombre, conformando la particular naturaleza humana. Asimismo, queda por señalar un tercer dualismo, hoy mentado como epistemológico, basado en la relación sujeto/objeto, ${ }^{21}$ en virtud de la cual se conforma un

\footnotetext{
${ }^{18}$ Esta imagen mecanicista de la naturaleza propuesta por Descartes, fue posteriormente adoptada por Newton y su modelo de ciencia moderna.

${ }^{19}$ Heinz Heimsoeth, La metafísica moderna (Madrid: Revista de Occidente, 1966).

${ }^{20}$ Phillipe Descola, Más allá de naturaleza y cultura (Buenos Aires: Amorrortu editores, 2012).

${ }^{21}$ La relación de conocimiento que funda la tradición racionalista ha sido cuestionada por el "enfoque enactivo" de Varela (y que Arturo Escobar retoma), quien postula la noción de enacción, que 
modelo de conocimiento en el que el sujeto cognoscente, constituido previo al acto de conocer, se dirige a un mundo que también preexiste al acto. Este binomio sujeto/objeto será fundamental para la construcción del conocimiento científico moderno y su pretensión de la objetividad.

Ahora bien, con Galileo ${ }^{22}$ la matematización del universo fue postulada de manera radical, tras sostener que este se encuentra escrito en lenguaje matemático. De tal modo, el universo se comprende como un sistema físico regulado por leyes capaces de ser expresadas en un lenguaje físico-matemático. Las cualidades que se tornan apreciables para esta nueva ciencia son aquellas que pueden ser cuantificables, las determinaciones cuantitativo-matemáticas de los cuerpos, tal es el caso de la relevancia que le otorga Galileo a las propiedades del movimiento en cuanto tal. Así, distinguía entre cualidades primarias y secundarias, a fin de retirar de los cuerpos todo "comportamiento" para dejarles únicamente el movimiento. En palabras de Bruno Latour: "[d]ejando de lado las cualidades secundarias -el color, el olor, la textura, pero también la generación, envejecimiento y la muerte - y aferrándose únicamente a las cualidades primeras — la extensión y el movimiento-, todos los planetas, todos los soles, todas las galaxias podrían ser tratados como bolas de billar". 23

A partir de ese momento, hay una universalización de las cualidades primarias de los cuerpos, por lo que basta conocer un cuerpo para conocer a todos, dado que todos funcionan bajo las mismas leyes mecánicas.

El resto de las cualidades de las cosas que nos son dadas por los sentidos escapa a todo conocimiento "claro y distinto", en términos cartesianos. De esta manera, todo proceso del mundo corpóreo se reduce a transmisiones mecánicas de movimiento determinadas por leyes rigurosas. La aplicación cada vez mayor de medidas cuantitativas

comprende la cognición como una acción encarnada. De esta manera se distancia de la relación sujetoobjeto en donde una conciencia preestablecida se dirige a conocer un mundo preestablecido, sobre el cual forma una representación. "La tradición racionalista Occidental, para la cual el 'mundo exterior' preexiste nuestras interacciones. En el enfoque enactivo siempre estamos inmersos en una red de interacciones que es, a cada instante, el resultado de nuestras historias biológicas y culturales. Necesariamente co-creamos el mundo con otros (humanos y no humanos), con quienes vivimos en coexistencia". Escobar, Autonomía y diseño, 103.

${ }^{22}$ Guillermo Boido, Noticias del planeta Tierra. Galileo Galilei y la revolución científica (Buenos Aires: AZ Editores, 1996).

${ }^{23}$ Bruno Latour, Cara a cara con el planeta (Buenos Aires: Siglo XXI Editores, 2017), 95. 
a fenómenos físicos de la Tierra. Asimismo, con Galileo la ciencia se piensa capaz de descubrir una verdad global, es decir, no sólo se trata de una naturaleza escrita en lenguaje matemático, sino que es un lenguaje único. En este universo homogéneo, la experimentación local descubre una verdad global. ${ }^{24}$

La distinción de las cualidades, señalada anteriormente, es relevante en cuanto fracciona el mundo entre una parte desanimada, declarada objetiva e inerte, y una "sobreanimada", subjetiva, consciente y libre. ${ }^{25}$ Así, tal escisión de cualidades contribuye al esquema ontológico naturaleza/cultura que posteriormente se consolida. De este modo, la ciencia moderna, desde sus inicios ha planteado una noción de la naturaleza muerta y pasiva, restringiendo la relación que el sujeto cognoscente tendría con ella, con la que se comunicaría únicamente para descubrir sus secretos, que lo harían dueño de ella. En el siglo XVII, entonces, como dirán algunos/as historiadores/as de la ciencia "hemos asistido a una transformación irreversible de nuestra relación con la naturaleza gracias a los éxitos de la ciencia moderna. En este sentido, se puede hablar de revolución científica". ${ }^{26}$

Otra de las figuras relevantes en la configuración de la ciencia moderna y la idea mecanicista de la naturaleza es el filósofo y científico Francis Bacon, quien inscribe un sesgo de dominación en el método científico. Fue uno de los referentes primordiales del empirismo inglés, asimismo, estableció parte de los fundamentos filosóficos de la revolución científica del siglo XVII. En una de sus obras más importantes, La gran restauración, ${ }^{27}$ introduce la distinción entre "anticipaciones de la naturaleza" e “interpretaciones de la naturaleza". Esta última es el tipo de aproximación que considera apropiada para el conocimiento de la naturaleza, facilitado por su método inductivo. Al igual que el proyecto cartesiano, pretendió la refundación de los cimientos del conocimiento y realizó una fuerte crítica al estado del conocimiento de su tiempo. Postuló un nuevo método para guiar correctamente la razón, como una cuestión necesaria a raíz de que "la lógica que hoy en día se emplea es inútil para la invención científica". ${ }^{28}$ En un

\footnotetext{
${ }^{24}$ Cfr. Ilya Prigogine e Isabelle Stengers, La nueva alianza. Metamorfosis de la ciencia (Madrid: Alianza Editorial, 2004).

${ }^{25}$ Cfr. Latour, Cara a cara, 104.

${ }^{26}$ Prigogine y Stengers, La nueva alianza, 32.

${ }^{27}$ Francis Bacon, Novum Organum (Buenos Aires: Editorial Losada, 2003).

${ }^{28}$ Ibid., 77.
} 
mismo sentido, pronunció que "las ciencias que por ahora poseemos no son otra cosa que disposiciones y arreglos de cosas encontradas antes; no métodos de invención ni fórmulas para nuevas producciones". ${ }^{29}$ En este fragmento se manifiesta una racionalidad de carácter instrumental, que será predominante en la ciencia moderna desde sus inicios.

Uno de los objetivos de aquella obra, además de restaurar el saber, es convertir al Hombre en amo y señor de la naturaleza gracias al conocimiento verdadero. Ello consistía en recuperar el lugar que tenía Adán antes de su caída y expulsión del paraíso (según el relato bíblico), el poder que se le había concedido sobre la naturaleza, haciendo patente la relación entre ciencia y religión bajo el precepto del antropocentrismo. ${ }^{30} \mathrm{Se}$ reitera la consideración del hombre como un observador separado en un mundo que le es ajeno, pero del que es dueño. Para Bacon, el científico debía torturar a la naturaleza hasta arrancarle sus secretos, "penetrar" 31 en lo más íntimo de la naturaleza para "extraerle" y "arrancarle" sus axiomas. Más allá de los principios metodológicos que establece para el conocimiento apropiado de la naturaleza, nos interesa señalar el lugar primordial de Bacon para legitimar una relación instrumentalista y de dominación con la naturaleza.

La imagen de una naturaleza autómata presente en la ciencia clásica (moderna) y en la ontología cartesiana, de carácter dualista, niega la complejidad de la physis y su devenir, asumiendo un mundo eterno y regido por leyes inmutables. ${ }^{32}$ Asimismo, se establece que las mismas son accesibles al hombre mediante la mecánica racional, por lo cual las leyes matemáticas pueden ser efectivamente descubiertas. Esto fue ejemplificado por la ciencia de Newton, quien "descubrió" una ley universal, a la que obedecen los cuerpos celestes y el mundo sublunar. Una misma ley que da cuenta al mismo tiempo de la caída de las piedras en el suelo y del movimiento de los planetas alrededor del sol. Con

\footnotetext{
${ }^{29}$ Ibid., 76.

${ }^{30}$ Una concepción similar aparece en John Locke sobre el Hombre como amo y señor de la naturaleza. $C f r$. John Locke, Segundo tratado sobre el gobierno civil (Barcelona: Alianza Editorial, 1994).

${ }^{31}$ Algunos estudios por parte de historiadoras feministas de la ciencia han señalado las metáforas de violación y tortura que aparecen en los escritos de Bacon y otros como Maquiavelo, que asientan las bases de la ciencia moderna clásica. Para ello Cfr. Sandra Harding, Ciencia y feminismo (Madrid: Ediciones Morata, 1996).

${ }^{32}$ Este punto puede enriquecerse con lectura que hace el filósofo español Sáez Rueda sobre los conceptos de natura naturans y natura naturata. La primera, como aquella potencia creadora que aún contenía la noción de physis griega, pero que en la modernidad y con el modelo científico, se le dará privilegio a la naturaleza como natura naturata. Cfr. Luis Sáez Rueda, El ocaso de Occidente (Barcelona: Herder, 2015).
} 
este acierto "la ciencia parecía demostrar que la naturaleza no es más que un sumiso autómata". ${ }^{33}$ A los ojos del siglo XVIII, Newton era el hombre que había descubierto el lenguaje que habla la naturaleza, como dice Ilya Prigogine e Isabelle Stengers, era "el 'Nuevo Moisés' a quien fueron mostradas las 'tablas de la ley",.34

Con la racionalidad newtoniana se recogen los elementos filosóficos que hemos ido señalando del siglo XVII, para contribuir al progresivo desencantamiento del mundo. Éste, sabemos, es uno de los procesos mediante el cual se identifica los inicios de la modernidad, dado que su descripción del mundo se reduce a la aplicación de leyes generales $\mathrm{y}$, de esta manera, concibe su funcionamiento como sometido a este esquema universal. Este mundo desencantado, acorde a la res extensa inanimada de Descartes, también se presenta como un objeto manejable, dado que aquel esquema, basado en la reducción de la diversidad del mundo mediante leyes generales, también opera como un sistema de control y dominación. El hombre que ha exteriorizado el mundo, extraño al mundo, se coloca también como su dueño.

\section{La ontología dualista moderna en su carácter naturalista}

En el apartado anterior dimos cuenta del modo en que la tradición cartesiana cristalizó la separación abismal entre el Hombre — sustancia pensante - y la naturaleza — sustancia extensa- En este marco, la única relación posible de establecer entre ambas sustancias es la de sujeto/objeto, es decir, exclusivamente una relación cognoscente en la que el sujeto tendrá potestad sobre el objeto. Respecto a esto último, señalamos la introducción de una pretensión de dominación, por lo que la modernidad por fin parece llegar a ubicar al Hombre como amo y señor de la naturaleza mediante la ciencia y la técnica. Es así que entre ambas sustancias no hay ningún otro vínculo entre sí del cual dependan sus existencias, son totalmente autónomas. Este dualismo metafísico (así como también, epistemológico y antropológico) se torna central para la modernidad porque permanecerá en los supuestos sobre los que se funda la episteme moderna, así como las formas

\footnotetext{
${ }_{33}^{33}$ Prigogine y Stengers, La nueva alianza, 34 .

${ }^{34}$ Ibid., 51.
} 
dominantes de organización política, social y económica. Por lo dicho, entonces, la ontología moderna occidental es, predominantemente, una ontología dualista.

Asimismo, en la ontología moderna subyace la creencia en la existencia de un ámbito propio de lo "natural", por lo que también se la denomina ontología naturalista, noción que permite poner la atención en tal creencia. La misma se acuña a partir de los aportes de Descola, antes nombrado en relación al Antropoceno, que ha realizado importantes contribuciones al llamado "giro ontológico" en las Ciencias Sociales y Humanas. El filósofo define al naturalismo como el modo de identificación dominante en occidente. ${ }^{35}$ Tal ontología se basa, principalmente, en la creencia de que la naturaleza existe en cuanto "ciertas cosas deben su existencia y desarrollo a un principio diferente del azar y de los efectos de la voluntad humana". ${ }^{36}$ En otras palabras, la singularidad del naturalismo radica en la conformación de un dominio ontológico específico, regido por la necesidad y el orden, en donde todo tiene una causa o una razón, ya sea ubicada en una instancia trascendente o inmanente al tejido del mundo, como las leyes de la naturaleza.

Desde el naturalismo nosotros, como humanos, compartimos con el resto de los entes que habitan el mundo, un cuerpo, una materialidad, en términos cartesianos, la res extensa. Lo que radicalmente nos distingue, diferencia y separa es la conciencia o la res cogitans y, posteriormente, también será la noción de "cultura". El naturalismo, como principio rector de nuestra ontología, se asumió como un supuesto "natural" privilegiado- que estructuró tanto la percepción de otras ontologías como nuestra construcción epistemológica.

Ahora bien, Descola en su libro Más allá de naturaleza y cultura realiza un recorrido histórico, epistémico y filosófico del modo en que se conformó paulatinamente la división entre naturaleza y cultura, propia de la ontología naturalista. Da cuenta de los complejos mecanismos que intervinieron en la emergencia de la

\footnotetext{
${ }^{35}$ La noción de "modo de identificación", refiere a la manera de definir las fronteras de sí mismo y del otro, la manifestación en las conceptualizaciones y el tratamiento de los humanos y los no-humanos. Cfr. Phillipe Descola, "La antropología y la cuestión de la naturaleza", en Germán Palacio y Astrid Ulloa, Repensando la naturaleza. Encuentros y desencuentros disciplinarios en torno a lo ambiental (Colombia: Universidad Nacional de Colombia-Sede Leticia, 2002).

${ }^{36}$ Ibid., 161.
} 
noción moderna de naturaleza y la nueva relación que establece el hombre con el mundo. Entre los elementos relevantes, se puntualiza en el surgimiento de algunos dispositivos técnicos que afectarán tanto al arte como a la ciencia, que mediarán el acercamiento del hombre moderno al mundo: la perspectiva lineal, el telescopio y el microscopio. En lo que compete al arte, en el Renacimiento el paisaje adquiere autonomía como temática en la pintura ${ }^{37}$ y se incorporan los estudios de la perspectiva lineal para su representación. Así, la perspectiva lineal pretende reconstituir el mundo como un espacio racional que escapa a las coacciones psicofisiológicas de la percepción $\mathrm{y}$, de esta manera, ocurre una "objetivación de lo subjetivo". ${ }^{38}$ Esto responde al particular mecanismo moderno en el que los fundamentos de la objetividad se encuentran en el sujeto.

Mediante esos dispositivos se va configurando una nueva relación entre el individuo y el mundo, entre quien mide con la mirada y un espacio sistematizado, reconstruido por reglas matemáticas. Al mismo tiempo que se crea una distancia entre el hombre y el mundo, es el primero quien sistematiza y organiza la exterioridad recién conquistada. "De tal modo, la perspectiva lineal instituye, en el ámbito de la representación, la posibilidad de ese cara a cara entre individuo y naturaleza que llegará a ser característico de la ideología moderna, y cuya representación artística será la pintura paisajística". 39

Tal representación del ambiente en su exterioridad por parte de la pintura paisajística no debe disociarse del movimiento de matematización del espacio que se efectúa en el siglo XVII por la geometría, la física y la óptica, y la filosofía cartesiana, proceso ya señalado. Éste es un periodo significativo que se desencadena desde el giro copernicano (el abandono de un paradigma geocéntrico) hasta la res extensa de Descartes. Allí opera una geometrización del espacio que concibe al mismo como un

\footnotetext{
${ }^{37}$ No desarrollaremos las relaciones entre la pintura paisajista y la noción de naturaleza que comenzaba a emerger en el Renacimiento, tampoco las múltiples tematizaciones que ha adquirido la naturaleza a lo largo de la historia del arte; sin embargo, comprendemos que radicaría un punto interesante a analizar.

${ }^{38}$ Sin embargo, allí radica una paradoja, la arbitrariedad de la mirada del observador es el punto de partida, es quien sistematiza el universo exterior y quien organiza esa exterioridad. Panofsky destaca esta paradoja y relaciona la invención de la perspectiva lineal con la emergencia de una nueva concepción del mundo. $C f r$. Erwin Panofsky, La perspectiva como 'forma simbólica' (Barcelona: Fábula y Tusquets Editores, 2003).

${ }^{39}$ Descola, Mas allá, 105.
} 
soporte inerte, anterior e independiente a cualquier objeto que se pose "sobre" él, así como también, se amarra a la idea de mundo como un receptáculo.

En este marco, la nueva relación con el mundo privilegia el sometimiento de lo real a la vista, habilitado por la invención de algunos dispositivos técnicos que hemos nombrado. Brevemente, recordemos que en 1609 Galileo muestra el primer telescopio astronómico registrado y, en 1590, Zacharias Janssen inventa el primer microscopio. Así, encontramos una predominancia del sentido de la vista, en detrimento de los otros. En consecuencia, como Descola manifiesta, "ahora muda, inodora e impalpable, la naturaleza se vació de vida. Olvidada la buena madre, desaparecida la madrastra, sólo quedaba el autómata ventrílocuo del cual el hombre podría mostrarse como dueño y señor" 40

Ese vacío de vida, que caracteriza a una naturaleza inanimada y muda, adquiere su máxima expresión en la imagen del mundo como una máquina cuyos engranajes pueden ser desmontados por los científicos, representada a partir de la revolución mecanicista del siglo XVII, como hemos explicitado. La construcción conceptual y científica de la naturaleza, también estuvo atravesada por constantes intentos de pensar al hombre en su excepcionalidad, a lo largo de la historia del pensamiento occidental canónico. Esta búsqueda de la singularidad, derivó en la afirmación de un privilegio ontológico conferido a la humanidad frente a otros seres, en otras palabras, a un férreo antropocentrismo. En ello, no podemos desconocer la incidencia de la operación llevada a cabo por la tradición judeo-cristiana, en la que el hombre no es un elemento más entre las obras de Dios, sino que tiene el derecho y la misión de administrar la Tierra en función de sus necesidades.

Esta visión antropocentrista sobre el mundo y la "naturaleza", permanecerá y se profundizará en la filosofía moderna, por ejemplo, en el pensamiento político de John Locke: "Dios, que ha dado en común el mundo a los hombres, también les ha dado la razón, a fin de que hagan uso de ella para conseguir mayor beneficio de la vida, y mayores ventajas. La tierra y todo lo que hay en ella le fue dada al hombre para soporte y comodidad de su existencia". ${ }^{41}$

En la filosofía política moderna, se hallan aspectos que forman parte de la configuración de la naturaleza en el marco del dualismo naturaleza/cultura, tales como

\footnotetext{
${ }^{40}$ Ibid., 108.

${ }^{41}$ Locke, Segundo tratado, 56.
} 
una concepción de la tierra como receptáculo, como propiedad del hombre y una comprensión antropocéntrica del valor (en cuanto la tierra por sí misma no posee valor).

Entonces, como se anunció, se inaugura en la modernidad occidental una nueva relación con el mundo, basada en el distanciamiento que el individuo moderno establece con la naturaleza, sometida ésta a leyes posibles de conocer y de manipular. En el interés por el conocimiento, se juega el interés por la dominación y el control. En este marco se asientan los cimientos ontológicos que desafectarán al ser humano del mundo que habita. Ante la pasividad de una naturaleza mecánica, emerge la posibilidad de desmontar sus engranajes y someterla a los fines del hombre, para finalmente emanciparse de ella. Así, el hombre moderno, inaugura el estado civil mediante el "contrato social", emblema de la filosofía política moderna, que explicita el quiebre: en adelante la ciudadanía será para el individuo único sujeto de derechos. Todo lo que queda del lado de la naturaleza o cercano a ella no admite agencia política, su participación es ilegítima. Este proceso alcanza su punto culminante en el siglo XIX con la conformación del dualismo naturaleza/cultura, cuando ambos términos se cristalizan como dominios autónomos, con sus respectivos programas metodológicos al interior de la episteme moderna (el dualismo metodológico explicación/comprensión). ${ }^{42}$

\section{La emergencia del mundo de la cultura: coronación de la ontología dualista}

En la época del racionalismo cartesiano, la naturaleza aún no se comprendía en una relación dicotómica con la cultura o con la sociedad, sin embargo, sí se diferenciaba de una "naturaleza humana". El surgimiento de las nociones de "sociedad" y de "cultura" datan de fines del siglo XIX y comienzos del siglo XX, momento caracterizado por las discusiones

\footnotetext{
${ }^{42}$ Esta particularidad de Occidente, ha sido temática central de muchos historiadores de la ciencia y filósofos. Por caso, algunas obras sobre la historia de la idea de la naturaleza, realizadas por Lenoble, Moscovici y Glacken. En este sentido, recordamos que el objeto de dar cuenta de algunos aspectos que han intervenido en la separación entre la naturaleza y el mundo humano, sin pretender hacer una exposición analítica y minuciosa, radica en destacar cómo se construye "un dispositivo ontológico de índole particular que sirve de basamento a la cosmogénesis de la modernidad" (Descola, Más allá, 110). Por lo cual, advertimos que la ontología naturalista se vuelve exótica y singular en comparación a otras distribuciones ontológicas que pueblan el (los) mundo(s) y ese movimiento epistémico es el que interesa en este trabajo.
} 
metodológicas sobre la explicación y la comprensión en torno a las Ciencias de la Naturaleza y las Ciencias del Espíritu (o de la Cultura). ${ }^{43}$ Paulatinamente, se cristalizó la división disciplinar de la episteme moderna, presente hasta nuestros días, entre las ciencias físico-naturales y las ciencias humanas y sociales, en la cual se manifiesta la escisión ontológica entre naturaleza y cultura. Se puede notar hasta aquí que la conformación del dualismo naturaleza/cultura es producto de un proceso específico, propio de la modernidad occidental. Esto es señalado por Descola, quien afirma la insuficiencia de proyectar este dualismo, propio de la cosmovisión moderna, en otras sociedades, como lo ha hecho la práctica científica, en particular la antropología tradicional.

Si indagamos sobre la noción de "cultura", encontramos diversas definiciones que orientan múltiples discusiones, principalmente, al interior de la Antropología. A continuación, se articularán diferentes acepciones para poder comprender cómo emerge la configuración de un nuevo ámbito ontológico que fortalecerá la separación del hombre de aquello que llamamos naturaleza. Es así que resulta pertinente extraer algunas cuestiones teóricas de dicha disciplina en tanto "el problema general de toda etnología es, de hecho, el de las relaciones (de continuidad o discontinuidad) entre la naturaleza y la cultura" ${ }^{44} \mathrm{y}$, por ende, tiene un rol relevante en la conformación del dualismo ontológico.

En primer lugar, una definición calificada de "humanista" considera a la cultura como el carácter distintivo de la condición humana. $\mathrm{Su}$ formulación canónica fue realizada en 1871 por Edward B. Tylor, quien definió la Cultura o la Civilización como el conjunto de los saberes, las creencias, el arte, las conductas, el derecho, las costumbres y cualquier otra disposición adquirida por el hombre cuando vive en sociedad. Esta noción, que fue adoptada por los antropólogos evolucionistas, ha sido central para la conformación del campo de la Antropología moderna y se acercó al uso predominante durante la Ilustración, cuando "cultura" aparecía como sinónimo de "civilización”. Cabe

\footnotetext{
${ }^{43}$ No debemos pasar por alto que la comprensión de la idea de cultura como independiente de las realidades naturales, se propaga en los debates de finales de siglo XIX en Alemania (entre los que intervienen Dilthey, Weber, entre otros) que apuntaban a precisar los métodos y los objetos respectivos de las ciencias de la naturaleza y las ciencias del espíritu (posteriormente, con Rickert, se hablará de ciencias de la cultura). "En un combate librado tanto contra la filosofía idealista de la historia como contra el naturalismo positivista, historiadores, lingüistas y filósofos se esfuerzan entonces por dar fundamento a la pretensión de las humanidades de convertirse en ciencias rigurosas, dignas del mismo respeto que imponen la física, la química o la fisiología animal" (Descola, Más allá, 128).

${ }^{44}$ Michel Foucault, Las palabras y las cosas (Buenos Aires: Siglo XXI, 1968), 366.
} 
hacer mención que, por su parte, “civilización" en la historia canónica del pensamiento moderno en ocasiones ha estado en relación opositiva con las nociones de "salvaje", "naturaleza" o "bárbaro". De manera predominante también se asociaba a una visión teleológica y evolucionista de la historia, en donde hay una fuerte prelación de la idea de progreso. Como afirma Quijano: “el mito fundacional de la versión eurocéntrica de la modernidad es la idea del estado de naturaleza como punto de partida del curso civilizatorio cuya culminación es la civilización europea u occidental". 45 Por lo que las ideas de "civilización" y "cultura" han estado históricamente asociadas a un modelo de cultura particular, el de la sociedad europea occidental.

En segundo lugar, siguiendo a Descola, ${ }^{46}$ referimos a la idea de Franz Boas, de principios del siglo XX, a partir de la cual la cultura abandona su formulación en singular al hablar de una multitud de realizaciones particulares. ${ }^{47}$ Es así que se vislumbra la idea de que cada pueblo posee una configuración única, de rasgos materiales e intelectuales sancionados por la tradición, con un modo de vida determinado, arraigado en la singularidad de una lengua. Las culturas aparecen como equivalentes en un cuadro sincrónico y se atiende a la riqueza de lo singular, rechazando un juicio evolucionista. La perspectiva boasiana asienta las bases de la orientación "culturalista" de la antropología norteamericana. Cabe señalar también que las raíces de este culturalismo norteamericano se hunden en el historicismo alemán. ${ }^{48}$

Una tercera concepción de cultura, que aún goza de actualidad, define a la misma como estructura simbólica y es postulada en la teoría semiótica de la cultura de Geertz, referente principal de la antropología simbólica del siglo XX. El modelo de Geertz

45 Aníbal Quijano, "Colonialidad del poder, eurocentrismo y América Latina", Edgardo Lander, La colonialidad del saber: eurocentrismo y ciencias sociales. Perspectivas latinoamericanas (Buenos Aires: CLACSO, 2000), 220.

${ }^{46}$ Descola se basa en un inventario crítico sobre las diversas definiciones de cultura, realizado por Alfred Kroeber y Clyde Kluckhohn, entre las cuales selecciona dos de las acepciones que retomamos en este apartado. Cfr. Alfred Kroeber y Clyde Kluckhohn, Culture: A Critical Review of Concepts and Definitions (Cambridge, MA: Harvard University Press, 1952).

${ }^{47}$ En un artículo Stocking analiza cómo Boas abandona el concepto de cultura tyloriano hasta crear una nueva idea, que triunfó y se implantó en la antropología a comienzos del siglo XX. Cfr. George W. Stocking, "Franz Boas y el concepto de cultura en perspectiva histórica", Race, Culture and Evolution. Essays in the History of Anthropology (Chicago: The University of Chicago Press, 1982).

${ }^{48}$ Cabe recordar que el historicismo alemán se apartó de la búsqueda de verdades universales para hacer hincapié en la inconmensurabilidad de los particularismos colectivos, los estilos de vida y las formas de pensamiento. 
propone una antropología más cercana a las ciencias humanas, cuya tarea primordial no es medir y clasificar (como el caso de una antropología de corte positivista) sino interpretar. Desde este punto, y dando continuidad al trabajo de Boas, admite la diversidad de "interpretaciones" del mundo (en tanto oficio hermenéutico) y de representaciones simbólicas. En otras palabras, la cultura es entendida como un sistema compartido de símbolos y significados convencionales y aprendidos, creados por el hombre, en virtud del cual, da significación a su existencia y le otorga un marco para orientarse en sus relaciones (con el mundo y consigo mismo). Desde esta concepción de cultura, la singularidad del hombre está dada por su capacidad de crear tramas de significado. En relación a esto, Geertz alude a su coincidencia con Weber: ${ }^{49}$ "[c] reyendo con Max Weber que el hombre es un animal inserto en tramas de significación que él mismo ha tejido, considero que la cultura es esa urdimbre y que el análisis de la cultura ha de ser, por lo tanto, no una ciencia experimental en busca de leyes, sino una ciencia interpretativa en busca de significaciones". 50

De esta manera, la dimensión de lo simbólico, del significado, de lo inmaterial y de lo connotativo, remite al plano propiamente humano, al mundo de la cultura escindido del mundo natural y material. No obstante, las diferencias y discusiones al interior de la disciplina antropológica, nos interesa señalar, la existencia de un horizonte de referencia común que excede a dicho ámbito. Éste se trata de una comprensión de la cultura, o las culturas, como un sistema de mediación con la "naturaleza", que ha sido invención de la humanidad y asignado como atributo distintivo de la misma. Asimismo, también encontramos la manifestación de esta concepción en el pensamiento político moderno, principalmente en su corriente contractualista, según el cual la sociedad civil se funda mediante un contrato social que separa a los hombres del "Estado de Naturaleza". En este sentido, desde la ontología moderna, además de tornar el mundo de la cultura como exclusivo de lo humano, junto con ella, también lo es el atributo de lo político y la participación concomitante en la sociedad civil.

\footnotetext{
${ }^{49}$ Cabe aclarar que, entre los múltiples mecanismos epistémicos que contribuyen a la separación moderna naturaleza/cultura, el caso de Weber, en el marco de la sociología comprensiva, es relevante dado que plantea una discontinuidad entre el ámbito de la naturaleza y el ámbito de lo social, este último como el ámbito de los sentidos.

${ }^{50}$ Clifford Geertz, La interpretación de las culturas (Barcelona: Editorial Gedisa, 2003), 20.
} 


\section{La distribución moderna de los ámbitos científico y político}

Luego del recorrido realizado sobre la compleja conformación del binomio naturaleza/cultura, es menester precisar el lugar predominante que tiene dicha separación para la "constitución moderna", como la llama Bruno Latour. ${ }^{51}$ Los aportes del filósofo, reconocido por sus trabajos en el ámbito de los estudios sociales de la ciencia y la tecnología y sus análisis sobre la modernidad y la crisis socio-ecológica actual, nos brindan elementos para comprender el alcance ontológico y político del dualismo. Cabe señalar que Latour también es un importante referente "giro ontológico", al igual de Descola, y ambos han realizado investigaciones críticas en torno al dualismo naturaleza/cultura. En el caso del primero, encontramos la propuesta de una nueva ontología posdualista para pensar la trama del presente. ${ }^{52}$ Descola, por su parte, ubica a la ontología naturalista como una ontología más entre otras, abriendo el juego a otras formas de pensar y hacer mundo.

Desde los análisis de Latour, la modernidad se constituye en tanto es capaz de diferenciar aquello que es naturaleza de lo que es cultura, afín a lo que hemos desarrollado como ontología dualista y naturalista. Son dos dualismos los que caracterizan la "constitución moderna", conformando las dos grandes divisiones, una interna y otra externa. La Gran División interna entre "naturaleza" y "cultura" explica la Gran División externa entre "nosotros" y "ellos"; en consecuencia, los modernos son los únicos que diferencian absolutamente entre naturaleza y cultura. "Todos los otros [...] no pueden separar realmente lo que es conocimiento de lo que es sociedad, lo que es signo de lo que es cosa, lo que viene de la naturaleza tal como es de lo que requieren sus culturas". ${ }^{53}$ Claro que todos tienen culturas o perspectivas del mundo, pero reconocer este hecho, es decir, reconocer que hay una realidad —en términos de "un mundo único"- y que hay cultura - como representación simbólica de esa realidad—, es la clave para comprender la singularidad de la ontología moderna.

Una de las hipótesis que sostiene Latour es que lo moderno involucra dos grupos de prácticas distintas y que para ser eficaces deben permanecer separadas. "El primer conjunto

\footnotetext{
${ }^{51}$ Bruno Latour, Nunca fuimos modernos (Buenos Aires: Siglo XXI, 2007).

${ }^{52}$ Cfr. Bruno Latour, Políticas de la naturaleza (Barcelona: RBA Libros, 2012).

${ }^{53}$ Ibid., 148.
} 
de prácticas crea, por 'traducción', mezclas entre género de seres totalmente nuevos, híbridos de naturaleza y cultura. El segundo, por 'purificación', crea dos zonas ontológicas por completo distintas, la de los humanos, por un lado, la de los no humanos, por el otro".54 Mantener separadas estas dos prácticas es lo que define a la modernidad, dado que, en cuanto se visualiza la coimplicación de ambas, se desmantela el relato moderno.

Con base en la ontología dualista, se distribuyen los diferentes ámbitos de la episteme moderna, en la que la política queda restringida a un espacio exclusivamente humano, mientras que las entidades no-humanas o "naturales" quedan desprovistas de agencia política, aspecto manifiesto en el pensamiento político canónico. La concepción mecanicista de la naturaleza ha invadido la manera de vincularnos con aquello que suponemos como "mundo natural", siendo un único mundo del que sólo puede hablar "con verdad" la ciencia.

La naturaleza moderna se conceptualiza como una entidad desprovista de agencia y, por ende, incapaz de intervenir como actor político, dado que la política moderna se funda como una dimensión humana por excelencia, lejos de la naturaleza y en dirección hacia la razón y la libertad. ${ }^{55}$ De esta manera, la ontología dualista, al sostener la separación entre el mundo natural y el mundo socio-cultural, distribuye las correspondientes divisiones de trabajo: mientras que de la primera se encarga la ciencia junto con sus expertos y expertas, la segunda es la esfera de las disputas políticas y representaciones culturales. Desde el régimen moderno, ciencia y política no se mezclan. ${ }^{56}$ La primera "simboliza la representación objetiva de la naturaleza, la segunda es la negociación del poder para representar a la gente frente al Estado". 57

Según lo expuesto, la idea del atributo político como propio de lo humano forma parte de los supuestos que fundan la política moderna o "razonable", como la llama Mario Blaser, ${ }^{58}$ intelectual referente del planteo de la ontología política. ${ }^{59}$ Esto hoy es discutido

\footnotetext{
${ }^{54}$ Ibid., 28.

55 Marisol de la Cadena, "Política indígena: un análisis más allá de "la política", Revista Red de Antropologías del Mundo, núm. 4 (enero, 2009): 8.

${ }^{56}$ Esta división entre ciencia y política remite a una disputa epistémica en el siglo XVII entre Robert Boyle y Thomas Hobbes. Véase Steven Shapin y Simon Schaffer, El Leviathan y la bomba de vacío (Bernal: Universidad Nacional de Quilmes: 2005).

${ }^{57}$ Ibid., 8.

${ }^{58}$ Cfr. Mario Blaser, “¿Es otra cosmopolítica posible?”, Anthropologica, 36, núm. 41 (2018).

${ }^{59}$ La propuesta de la ontología política, de la que participan Mario Blaser, Marisol de la Cadena y Arturo Escobar, puede comprenderse también como parte del "giro ontológico" de las Humanidades, ya nombrado a propósito de Descola y Latour.
} 
desde diferentes espacios epistémicos, tanto desde orientaciones académicas ${ }^{60}$ como desde ciertas comunidades indígenas, afrodescendientes y campesinas en América Latina, las que defienden sus territorios como mundos relacionales. ${ }^{61}$ Desde allí, se problematiza la ontología política moderna por caso, ante la emergencia de ciertos conflictos en los que la presencia de "personas no-humanas" en política se vuelve central, al tiempo que la comprensión desde el marco conceptual de la política moderna se torna insuficiente. Además, la imposibilidad de seguir sosteniendo una imagen de la naturaleza como desanimada, se evidencia ante la intrusión de Gaia, acontecimiento trabajado por Stengers. ${ }^{62}$ Así, aquellas entidades que en principio son consideradas inanimadas e inertes en el entramado de las ontologías relacionales — por ejemplo, una laguna - participan activamente de lo social. Esta presencia pone en tensión la separación entre cultura y naturaleza, entre Hombre y Naturaleza.

\section{Líneas de cierre-apertura: de culturas a ontologías múltiples}

Ahora bien, llegados a este punto interesa ejercitar un cierre no-conclusivo que funcione como apertura y que dé cuenta de las continuidades posibles, puesto que la espesura temática imposibilita un cierre hermético y definitivo. Hasta el momento hemos dado cuenta del modo en que paulatinamente se conformó el dominio de la naturaleza, por un lado, y el de la cultura, por otro, en una relación de oposición propia de la ontología

\footnotetext{
${ }^{60}$ Es posible nombrar, a modo de ejemplo, algunos virajes posdualistas provenientes de ámbitos académicos que problematizan la ontología moderno-colonial y proporcionan insumos teóricos que habilitan un pensamiento relacional. En primer lugar, las discusiones en torno a la llamada "cosmopolítica", propuesta por la filósofa belga Isabelle Stengers, cuya incorporación brinda una novedosa manera de pensar y hacer política que no se enmarca en separación naturaleza/cultura. En segundo lugar, las últimas propuestas de Donna Haraway, que abonan a un feminismo multiespecie como respuesta a la lógica de devastación de la modernidad capitalista y a su antropocentrismo e individualismo.

${ }^{61}$ Ejemplo de ello son ciertas comunidades afrodescendientes y campesinas del Norte del Cauca en Colombia y el Pacífico colombiano, las comunidades indígenas de la región andina de América Latina, las comunidades autónomas en Chiapas, Michoacán y Oaxaca en México, las comunidades en el Amazonas, entre muchos otros. Todas ellas si bien mantienen conexiones parciales con la ontología moderna, son mundos en movimiento por la defensa de sus territorios y están involucrados en lo que Escobar llama "activación política de la relacionalidad". Asimismo, la lógica relacional nutre prácticas políticas como las asambleas ciudadanas socio-ambientales permanentes, los feminismos comunitarios y territoriales, activismos vinculados a guardianes y casas de semillas, a la soberanía alimentaria, etcétera.

${ }^{62}$ Cfr. Isabelle Stengers, En tiempos de catástrofe (Buenos Aires: Futuro Anterior / Ned Ediciones, 2017).
}

www.enclavesdelpensamiento.mx

https://doi.org/10.46530/ecdp.v0i30.422 
dualista. Este proceso, que se desenvolvió en la modernidad occidental, conforma una especificidad histórica difícil de extrapolar a otros escenarios, aunque las prácticas eurocéntricas de las Ciencias Sociales ${ }^{63}$ y, en particular, de las etnografías clásicas se han caracterizado por abordar, mediante esas categorías, otras conformaciones culturales otras ontologías - que escapan a las formas dominantes de concebir la "naturaleza".

Quizás podríamos admitir que hay diferentes concepciones de lo que es la naturaleza en función de la cultura en la que nos ubiquemos; sin embargo, un análisis que desmantele la geopolítica del conocimiento daría cuenta que por debajo del reconocimiento de la multiplicidad cultural opera una diferencia de poder. Desde el giro decolonial, esto refiere al despliegue de la lógica de la colonialidad, según la cual, la diferencia se manifiesta como la imposición de un conjunto de normas culturales como universales, ocluyendo y minusvalorando otras formas de saber, ser y hacer, otras ontologías.

Como desarrollamos, tales formas dominantes aluden a una lógica dualista, con raíces en el pensamiento cartesiano, que funda una comprensión de la subjetividad moderna como ajena e independiente al mundo físico-natural. Asimismo, bajo esa ontología, dicho mundo físico interesa en cuanto funciona como una máquina, regulable, predecible, manipulable. En este sentido, finalmente la modernidad capitalista nos deja ante una naturaleza inanimada, sin vida y de la que el humano no forma parte. La desafectación del sujeto moderno respecto del resto de los mundos no-humanos, la devaluación de los mundos que ha ubicado como "cercanos" a lo natural, alejados de lo civilizado, ha habilitado prácticas de subordinación, explotación, apropiación e invisibilización.

Es relevante explicitar que el análisis de este artículo involucra un desplazamiento epistémico al comprender, en clave ontológica y no culturalista, el modo particular de vinculación entre los humanos y los no-humanos que establece la ontología moderna. Es decir, si bien son premisas que asumimos en nuestro habitar, también se manifiestan en prácticas, al tiempo que habilitan y legitiman modos de vinculación.

Finalmente, consideramos que, habida cuenta del recorrido conceptual, indagar en modos de habitar no-capitalistas que respondan a la crisis multidimensional a la que asistimos implica un desplazamiento ontológico. Éste no sólo debe fisurar la ontología

\footnotetext{
${ }^{63}$ Sobre el eurocentrismo en las ciencias sociales y la colonialidad del saber, ver Lander (2000).
} 
moderna que se ha mostrado como la única opción posible, en su carácter totalizante, sino también poder visualizar y habitar otros modos de ser, otras ontologías. De esta manera, dejamos abierta la afirmación de que es posible encontrar claves para resistir y reexistir en ontologías posdualistas y relacionales, ${ }^{64}$ que comprendan las vinculaciones entre humanos y no-humanos en términos de interdependencia. Apostar a una relacionalidad radical, en la que nos comprendemos interexistiendo en un tejido complejo con otros seres, tiene una potencialidad política que es menester indagar.

64 Entiéndase por ontologías políticas relacionales, ontologías no-dualistas, ontologías no-modernas, ontologías planas, ontologías múltiples, entre otras, aquellas formas de concebir, habitar y construir el mundo que no sostienen una lógica binaria moderna/colonial, sino que vivencian una relacionalidad profunda e interdependencia entre heterogéneos mundos humanos y no-humanos. Estas ontologías otras, han sido excluidas de la retórica de la modernidad, pero siguen existiendo en diversas prácticas y comunidades (véase nota 61). Asimismo, la temática de las ontologías no-dualistas y las críticas a los dualismos modernos está cobrando importancia en debates al interior de las Ciencias Sociales y Humanas en las últimas décadas, ante insuficiencia de la ontología dualista moderno/colonial para orientarnos hacia futuros más vivibles (véase nota 60). 


\section{Bibliografía}

Bacon, Francis. Novum Organum. Buenos Aires: Editorial Losada, 2003.

Blaser, Mario. “¿Es otra cosmopolítica posible?”. Anthropologica, 36, núm. 41 (2018): 117-144.

Boido, Guillermo. Noticias del planeta Tierra. Galileo Galilei y la revolución científica. Buenos Aires: AZ Editores, 1996.

Borsani, María Eugenia. "El encuentro con la decolonialidad desde este sur". En Ejercicios decolonizantes en este sur. Buenos Aires: Ed. del Signo / Duke University, 2014.

De la Cadena, Marisol. "Política indígena: un análisis más allá de 'la política"'. Revista Red de Antropologías del Mundo, núm. 4 (enero 2009): 139-171.

Descartes, René. El discurso del método. Madrid: Gredos, 2011.

Descartes, René. Los principios de la filosofía. Barcelona: Alianza Editorial, 1995.

Descartes, René. Meditaciones Metafísicas. Buenos Aires: Prometeo, 2009.

Descola, Philippe. "La antropología y la cuestión de la naturaleza". En Germán Palacio y Astrid Ulloa, Repensando la naturaleza. Encuentros y desencuentros disciplinarios en torno a lo ambiental. Colombia: Universidad Nacional de Colombia, 2002.

Descola, Philippe. Más allá de naturaleza y cultura. Buenos Aires: Amorrortu Editores, 2012.

Escobar, Arturo. "Mundos y conocimientos de otro modo". Revista Tabula Rasa, núm. 1 (enero-diciembre 2003): 51-86.

Escobar, Arturo. "Cultura y diferencia: la ontología política del campo de Cultura y Desarrollo". Wale'keru. Revista de investigación en Cultura y Desarrollo, núm. 2 (2012): 7-16.

Escobar, Arturo. Autonomía y diseño: la realización de lo comunal. Popayán: Editorial Universidad del Cauca, 2016.

Foucault, Michel. Las palabras y las cosas. Buenos Aires: Siglo XXI, 1968.

Geertz, Clifford. La interpretación de las culturas. Barcelona: Editorial Gedisa, 2003. 
Heimsoeth, Heinz. La Metafísica Moderna. Madrid: Revista de Occidente, 1966.

Kearney, Hugh. Orígenes de la Ciencia Moderna 1500-1700, Madrid: Ed. Guadarrama, 1970.

Latour, Bruno. Cara a cara con el planeta. Buenos Aires: Siglo XXI, 2017.

Latour, Bruno. Nunca fuimos modernos. Ensayo de antropología simétrica. Buenos Aires: Siglo XXI, 2007.

Locke, John. Segundo tratado sobre el gobierno civil. Barcelona: Alianza Editorial, 1994. Mignolo, Walter. Desobediencia epistémica. Retórica de la modernidad, lógica de la colonialidad y gramática de la descolonialidad. Buenos Aires: Ed. del Signo / Duke University, 2010.

Mignolo, Walter. Habitar la frontera. Ciudad Juárez: CIDOB y UACJ, 2015.

Prigogine, Ilya y Isabelle Stengers. La nueva alianza. Metamorfosis de la ciencia. Madrid: Alianza Editorial, 2004.

Quijano, Aníbal. "Colonialidad del poder, eurocentrismo y América Latina”. En Edgardo Lander, La colonialidad del saber: eurocentrismo y ciencias sociales. Perspectivas latinoamericanas. Buenos Aires: CLACSO, 2000.

Quijano, Aníbal. “Colonialidad y modernidad-racionalidad”. En Zulma Palermo y Pablo Quintero, Aníbal Quijano. Textos de fundación. Buenos Aires: Ed. del Signo / Duke University, 2014. 